Historia y comunicación social

ISSN: $1137-0734$

\title{
Proyectos documentales digitales para la reconstrucción de la Historia de las mujeres con análisis de género ${ }^{1}$
}

\author{
María Adelina Codina-Canet²; Rosa San Segundo ${ }^{3}$
}

Recibido el: 24 de octubre de 2019 / Aceptado: 28 de septiembre de 2020

Resumen. Se recopilan los proyectos documentales cuyo objeto es recuperar la memoria de las mujeres y la reconstrucción de la Historia con perspectiva de género se proporciona información sobre ellos. Algunos proyectos están basados en técnicas de creación de fuentes de historia oral e incorporan medios tecnológicos para su realización y comunicación. Los proyectos, desde la perspectiva de análisis, rescatan la experiencia femenina como protagonista de la historia. La mayor parte de los proyectos están realizados por grupos de investigación en las universidades, a los que se suman iniciativas desarrolladas en el ámbito privado, proyectos realizados por organizaciones feministas, por fundaciones culturales de los sindicatos obreros y, en menor medida, por organismos públicos. El objeto es recuperar fuentes y preservar la memoria colectiva de las mujeres en el período histórico durante la transición española al sistema democrático en el último tercio del siglo XX.

Palabras claves: Archivo digital; análisis de género; fuentes históricas; fondo documental; fuentes orales; Organizaciones feministas.

\section{[en] documentary projects for the reconstruction of History with gender analysis}

\begin{abstract}
They compile and provide information on archival documentary projects, whose purpose is to recover the memory of women, and the reconstruction of History with a gender perspective. Some projects are based on techniques of creating oral history sources, and incorporate technological means for their realization and dissemination. The projects, from the analysis perspective, rescue the female experience as protagonists of the history, most of the projects are carried out by research groups, in the universities. In addition, initiatives developed in the private sphere, projects carried out by feminist organizations, cultural foundations of labor unions, and to a lesser extent by public bodies are added. The purpose is to create historical sources, and to preserve the collective memory of women in the historical period, of the political process of the transition to the democratic system. On the other hand, a project is proposed in order to protect archival documentation, documentary heritage, to preserve the memory of the feminist movement, we proceed to analyze the keys tending to execute a virtual archive project, in the digital age.
\end{abstract}

Keywords: Digital archive; documentary background; Feminist organizations; gender analysis; historical sources; oral sources.

Sumario: 1. Introducción. 2. Objetivos y metodología. 3. Resultados. 3.1. Proyectos documentales de memoria e historia de las mujeres. 3.1.1 Proyectos del Seminario de Fuentes Orales. 3.1.2 Proyectos de la Universidad Nacional de Educación a Distancia. 3.1.3. Proyecto Diccionari Biogràfic de Dones. 3.1.4. Proyecto Álbum de Mulleres. 3.2. Proyectos de memoria oral y digital sobre feminismo organizado en la transición. 3.2.1. Proyecto Entrevistas realizadas a mujeres. 3.2.2. Proyecto La Asamblea de Mujeres de Granada: 35 años de lucha feminista en la ciudad de Granada. 3.2.3. Proyecto Entrevistas a mujeres vinculadas a la asociación Ca la Dona. 3.2.4. Proyecto Archivo Oral del Sindicalismo Socialista-Mujeres. 3.3.5. Proyecto Biografías obreras y militancia sindical en las Comisiones Obreras. 3.2.6. Proyecto Archivo de Fuentes Orales para la Historia Social de Asturias. 3.2.7. Proyecto Memoria del Feminismo en el País Valenciano, 1970-1997. 3.2.8. Proyecto Una Transición en femenino. 3.2.9. Proyecto Haciendo historia: Género y Transición. 3.2.10. Proyecto Mujer y Memoria. 3.3. Proyectos digitales para el estudio del movimiento feminista de la transición. 3.3.1 Proyecto Centro de documentación digital Maite Albiz. 3.3.2 Proyecto Recuperación, preservación y difusión del legado histórico documental del movimiento feminista y de mujeres. 3.3.3 Proyecto Archivo de Feminismos de León. 3.3.4. Proyecto Carteles de la Biblioteca de Mujeres. 3.3.5. Proyecto Archivo de la Transición. 4. Conclusiones. 5. Referencias bibliográficas.

Cómo citar: Codina-Canet, M. A.; San Segundo, R. (2020) Proyectos documentales digitales para la reconstrucción de la Historia de las mujeres con análisis de género, Historia y comunicación social 26(1), 279-289.

Este artículo se enmarca en el proyecto identificado por RTI2018-093599-B-100 MCIU/AE/FEDER,UE. Título del proyecto: Historia, memoria y sociedad digital. Nuevas formas de transmisión del pasado reciente: la Transición a la democracia en España. Incluido en el Programa Proyectos I+D+I retos Investigación, del Programa Estatal I + D + I orientada a los Retos de la Sociedad, para el período 2019-2021

2 Universidad Carlos III de Madrid

E-mail:mcodina@db.uc3m.es

ORCID: https://orcid.org/0000-0002-4202-8816

3 Universidad Carlos III de Madrid

E-mail: rosa.sansegundo@uc3m.es

ORCID: https://orcid.org/0000-0002-1449-8175

Hist. comun. soc. 26(1) 2021: 279-289 


\section{Introducción}

La idea de recopilar proyectos documentales de la Historia de las mujeres y del feminismo español en línea va a permitir conformar, a modo de guía de información, un repertorio documental de fuentes al alcance de los usuarios, el personal investigador y los servicios de información de las bibliotecas y centros de documentación. En este trabajo, se presentan fuentes de información en forma de recursos web, con el objeto de dar respuesta a los usuarios interesados en obtener información sobre las mujeres españolas como protagonistas de los procesos históricos recientes.

Los proyectos de memoria de las mujeres realizados en España, básicamente, crean fuentes orales que testimonian los hechos históricos mediante relatos de vida de las mujeres entrevistadas. De igual forma, se crean recursos educativos para divulgar la historia de las mujeres y de activistas feministas, durante la Transición política a la democracia. Esta recopilación de proyectos documentales, desde un análisis de género, son fuentes primarias que sirven para el estudio del protagonismo de las mujeres y sus organizaciones. Son escasos los proyectos documentales que recuperan la documentación de archivo de las organizaciones feministas, para digitalizarla y ponerla a disposición de la investigación.

Al tratarse de la historia reciente de 40 años, algunas activistas y protagonistas han publicado su notoria contribución en memorias, biografías y relatos de vida de los sucesos vividos del pasado postfranquista y predemocrático del colectivo feminista. Igualmente, se han realizado proyectos de memoria para la historia de las mujeres, sin embargo, es manifiesta la nula presencia oficialista del protagonismo de las mujeres en el relato histórico y en los libros de texto.

En los últimos años surgen algunas iniciativas en pro de la memoria histórica de las mujeres, como la revisión de las fuentes documentales de archivo y estudio de la situación del caudal documental generado a partir de la lucha del movimiento de mujeres desde la Transición democrática española. Las investigadoras Codina-Canet y San Segundo (2016) afirman que España no ha conseguido que las organizaciones y las activistas feministas depositen los documentos de su actividad en centros de archivo para su custodia. Por tanto, existe el riesgo de desaparición de muchos de estos fondos documentales, como consecuencia de la naturaleza efímera tanto de los documentos como de los colectivos que no hayan prosperado.

La recuperación de la historia de las mujeres se enmarca en un nuevo modelo de representación, en el cual se combina el binomio Historia-Memoria, cuyo objetivo es, a través de las fuentes documentales, la reconstrucción y confrontación para contrastar la historia oficial, cuestionar las formas, objetar las carencias de los contenidos de la enseñanza reglada obligatoria y discutir la transmisión de la cultura en general (González Quintana, 2009; Brancas, 2015; Egido León y Eiroa San Francisco, 2017).

\section{Objetivos y metodología}

La categoría crítica de género, como herramienta de análisis, conduce a considerar género, en tanto que alude a la relación dialéctica de los sexos. Este trabajo que se presenta entraña el estudio de género como categoría crítica, y rescata la experiencia femenina en el relato histórico. El elemento de análisis son los proyectos que incorporan los contenidos o resultados de sus investigaciones en sus archivos en línea, los proyectos de carácter memorialista fundamentales en el protagonismo de las mujeres y del movimiento feminista, en los cuales se hace imprescindible la visión especializada sobre archivos, sociedad, memoria y las nuevas tecnologías aplicadas, con el objeto de vincular el análisis de la evolución de las fuentes para la investigación de la historia del movimiento feminista reciente, en relación con las nuevas tecnologías (Alberch y Cruz Mundet, 1999; Shaheed, 2014; González Quintana, 2009; Brancas, 2015).

Los objetivos trazados son conocer el estado de la cuestión de proyectos documentales y de proyectos de digitalización de archivos privados de las organizaciones feministas en España. La recopilación de proyectos memorialistas de recuperación documental de la historia de las mujeres es una tarea justificada con el objeto de obtener nuevas fuentes de información, que cuestionen la cultura transmitida a la sociedad. Una cultura no inclusiva y que excluye el papel de las organizaciones feministas en la historia de la Transición española. Así pues, se cuestiona la "cultura del olvido impuesta" a las organizaciones feministas y la exclusión de su participación política en la sociedad en el período de la Transición política a la democracia, en la "historia oficial"

De la toma de conciencia de la necesidad de recuperar la memoria de las mujeres surgen proyectos de memoria en buena parte de la geografía española y son notorios los realizados en ámbito académico de las universidades. Las universidades son espacios propicios donde emergen los proyectos tanto colectivos como individuales y liderados por grupos de investigación feminista, como seminarios e institutos de investigación, que emergen desde la década de los años ochenta. Los coloquios, encuentros, jornadas y congresos, cuya temática es la investigación histórico social de las mujeres y su difusión en forma de actas de congreso y artículos de revista, se enmarcan en el contexto de manifestaciones y ejercicios para recuperar la memoria colectiva. 
Los proyectos de memoria de las mujeres recopilados abordan trabajos relacionados con los archivos y sus documentos, con las fuentes de información y, en ocasiones, sus resultados son de naturaleza instrumental y sirven como herramienta para otras investigaciones. En algunos de los proyectos de recuperación de la memoria, el protagonismo no recae de manera exclusiva en las mujeres, sino que las mujeres se presentan como protagonistas junto a los hombres.

Más específicos son los proyectos de memoria e historia oral y digital realizados sobre las mujeres partícipes de la Transición democrática. Proyectos realizados en el ámbito universitario y también las propias organizaciones feministas y en las áreas de la mujer de los sindicatos obreros más representativos.

Si bien, algunos proyectos recopilados son de origen extra-universitarios, las académicas y las investigadoras del feminismo teórico, vinculadas a las universidades participan, de alguna manera, como asesoras científicas. Los proyectos de referencia de memoria de las mujeres incorporan medios tecnológicos, representados en archivos de testimonios orales, consistentes en grabaciones de audio y audiovisuales, y en sus transcripciones. Consecutivamente, tras el avance de la tecnología en la comunicación, se difunde este contenido en páginas web, en formatos electrónicos y digitales, posibilitando su difusión.

\section{Resultados}

\subsection{Proyectos documentales de memoria e historia de las mujeres}

Los primeros proyectos, recopilados de grupos de investigación, se circunscriben en la década de los años ochenta; su evolución ha sido favorable, en el sentido de la adaptación a las nuevas tecnologías, ya que, en varios casos, se trata de proyectos sectoriales sin conexión entre ellos. En la sociedad del conocimiento, se incorpora una nueva representación de la realidad derivada del impacto de las nuevas tecnologías en la historiografía, donde aparecen nuevas comunidades discursivas, donde la sociedad interactúa en el discurso de la historia y la memoria, incorporándose las humanidades participativas y digitales. En el siglo XXI, paralelo al rigor académico, emergen proyectos que influyen en la transmisión de los valores y del conocimiento en la sociedad, generando nuevas fuentes de información y comunicación que no se pueden soslayar, en la cual la realidad digital marca el nuevo compás en la evolución de los discursos.

\subsubsection{Proyectos del Seminario de Fuentes Orales}

Entre los primeros proyectos documentales de historia de las mujeres en España, se encuentra el Seminario de Fuentes Orales (SFO), un grupo de investigación constituido en asociación en el año 1984, que surge al amparo de la profesora del Departamento de Historia Contemporánea, María del Carmen García-Nieto en la Universidad Complutense, con el objeto de "desarrollar proyecto de investigación histórica del Tiempo Presente, a partir de la creación y potenciación de las fuentes orales, indispensables para la misma"(Seminario de Fuentes Orales, s.f.a). Además, para recuperar la memoria colectiva y "obtener de esta forma un corpus de información indispensable para la Historia del Tiempo Presente (Segunda República, Guerra Civil, Franquismo, Transición...)" (Seminario de Fuentes Orales, s.f.a).

Los proyectos de investigación histórica del Seminario SFO estuvieron dirigidos a testimoniar a las mujeres como sujetos de la historia española. Al menos, se materializaron el proyecto en torno a mujeres madrileñas activistas durante la guerra, proyecto titulado Mujeres en Madrid durante la Guerra Civil, desarrollado entre 1982-1988, se realizaron 41 entrevistas a milicianas, entre ellas, Dolores Ibárruri; el proyecto sobre mujeres pertenecientes a estratos sociales obreros en la capital, titulado Capas populares y urbanismo. Palomeras, un barrio obrero durante el franquismo, 1950-1980, en el cual se realizaron 33 entrevistas, que recogen el testimonio de la población de la periferia del barrio de Palomeras de Madrid, situando a las capas populares como sujeto de la investigación (Seminario de Fuentes Orales, s.f.b). Y otro proyecto del Seminario SFO, abarcó el estudio de la escuela en tiempos del franquismo, bajo el título de La Escuela franquista, 19381957, desarrollado entre los años 1991 y 1992, recopilándose en torno a 24 testimonios. La temática de la situación de la clase trabajadora en tiempos de la Transición política, proyecto denominado Conflictos obreros y Transición política. El caso de Madrid, 1975-1977 fue otro de los ejes de trabajo del Seminario, desarrollado durante el año 1993, que incluye 33 entrevistas a líderes sindicales y obreros de distintos sectores relacionados con las movilizaciones obreras durante el franquismo. Por último, la investigación sobre mujeres urbanas, proyecto nombrado Trabajo, cultura e identidad personal de las mujeres en un espacio urbano. Madrid 19501980 , en este proyecto se llegaron a realizar 21 entrevistas que testimoniaron la incorporación de la mujer al mundo laboral. Los proyectos fueron difundidos en una página Web, al menos desde los años 2010 a 2016, en tanto que, el material de estos proyectos orales está depositado en la Universidad Complutense y una copia en el Centro Documental de la Memoria Histórica de Salamanca (Seminario de Fuentes Orales, s.f.b). 


\subsubsection{Proyectos de la Universidad Nacional de Educación a Distancia}

Otros proyectos documentales, basados en el enfoque de crear recursos educativos, sobre la historia de las mujeres, son los auspiciados desde la Universidad Nacional de Educación a Distancia (UNED.), combinando el formato audiovisual. El resultado es una fuente de información para el estudio y la enseñanza. En el documento Historia crítica del Feminismo español (2) El Feminismo en la Segunda República, se traslada un índice de contenido con los 42 títulos de programas educativos presentados en series de televisión (UNED, 2013). Los proyectos de televisión empezaron a editarse en el año 1996, la primera producción propuesta es Vida y Obra literaria de Emilia Pardo Bazán, en la que se aborda la literatura de la escritora feminista; dos años después dio comienzo la serie nombrada Las mujeres en el mundo (UNED, 1998) comprende cuatro capítulos; algunas series continúan abiertas, la denominada Derechos de la Mujer y equidad de género (UNED, 1998 - 2017) abarca veinte años de producción, desde 1998 a 2017. Esta colección audiovisual, de más de cincuenta capítulos, analiza los aspectos de las mujeres y su posición social como sujeto en la historia, la violencia de género, el derecho al voto, mujeres en la república, mujeres en las cárceles franquistas, mujer y centros penitenciarios, es una de las colecciones con mayor continuidad en el tiempo. Y, desde la misma perspectiva de análisis, la colección bajo el título de Mujeres intelectuales. Mujeres en ciencia incluye varias facetas científicas del trabajo de las mujeres, en total dieciocho capítulos editados durante 2004 (UNED, 2004). Con posterioridad en el año 2012, dentro del proyecto audiovisual, se realizó la colección de reportajes Historia crítica del feminismo español; el denominado Las intelectuales de la Edad de Plata y, el llamado El camino de la mujer hacia la igualdad civil, del año 2007. Además, desde la Universidad Nacional de Educación a Distancia (UNED) se han emitido numerosos programas de radio, que desarrollan unidades didácticas tratando la condición y la historia de las mujeres, dirigidos a la educación y difusión del conocimiento.

\subsubsection{Proyecto Diccionari Biogràfic de Dones}

El proyecto de historia sobre la contribución de las mujeres en la sociedad, Diccionari Biogràfic de Dones, DBD, es el proyecto de reseñas biográficas creado desde la Red de Universidades Vives, la Generalitat de Catalunya y el Consell de Mallorca, con el objetivo de visibilizar la contribución de las mujeres, en los distintos estamentos de la sociedad y los acontecimientos de la historia de los territorios de habla catalana. Diccionari Biogràfic de Dones se desarrolla en la universidad, se trata de un proyecto dirigido por la investigadora medievalista Teresa Vinyolas Vidal, adscrita al Centro de Investigación de Mujeres de la Universidad de Barcelona, DUODA de la Universitat de Barcelona y en el ámbito de la biblioteconomía y archivística por la profesora Nuria Jornet Benito. La base de datos del Diccionari Biogràfic de Dones contiene más de seiscientas biografías de mujeres de diferentes campos del conocimiento y ámbitos de actuación abarca desde el siglo I al XXI, "el fons actual de DBD inclou 655 biografies de dones de diferents camps del coneixement, i àmbits d'actuació, de Catalunya, el País Valencià, les Illes Balears, Catalunya nord, Andorra i Sardenya, en un arc cronològic que abarça del segle I al segle XXI" (Xarxa Vives d' Universitats, s.f.).

\subsubsection{Proyecto Álbum de Mulleres}

Otro proyecto documental ajeno a la universidad es el nombrado Álbum de Mulleres fue impulsado y coordinado por la Comisión de Igualdad del Consejo de Cultura Gallega, con el objeto de recuperar la memoria histórica de las mujeres gallegas. Es un proyecto de centro de documentación digital para divulgar e investigar la contribución de las mujeres en la sociedad y cultura gallega, en todas las épocas, actualmente contiene una galería biobi-bliográfica de 140 gallegas. El proyecto Álbum de Mulleres activa, entre otros proyectos, el denominado Recuperación da documentación e memoria do Movemento Feminista Organizado en Galicia, a través de espacios web desde el año 2005 y una colección especial de publicaciones, han editado materiales sobre fuentes documentales y de información de archivos feministas. El informe publicado durante el año 2013, coordinado por la especialista en antropología feminista social Mariam Mariño Costales y la investigadora feminista y profesora de Universidade da Coruña Carmen Pérez Pais Recuperación da documentación e memoria do Movemento Feminista Organizado en Galicia ha identificado el estudio de grupos, colectivos, organizaciones y librerías de mujer y fuentes bibliográficas y documentales (Pérez Pais y Mariño Costales, 2013). Uno de los temas monográficos radica sobre la despenalización del aborto en Galicia, el Aborto Corenta anos de mobilizacións, con acceso a documentos históricos de la lucha feminista por la legalización del aborto en Galicia. Los documentos han sido recuperados provenientes de las siguientes asociaciones Asociación Galega da Muller, Asamblea de Mulleres da Coruña, Movimento Feminista Galego, Coordinadora feminista de Vigo, Coordinadora Feminista de Galicia (Álbum de mulleres, s.f. a). 


\subsection{Proyectos de memoria oral y digital sobre feminismo organizado en la transición}

Los proyectos de memoria circunscritos al período de la transición son intentos de preservar la memoria histórica de las mujeres en este período. Parten de la necesidad de recuperar la historia feminista, y, en contados casos, la documentación generada, al objeto de visibilizar la aportación de las mujeres organizadas en los cambios políticos y sociales acontecidos en España. De manera que algunos proyectos de memoria del movimiento feminista, básicamente, crean fuentes orales que testimonian los hechos históricos mediante relatos de vida de las mujeres entrevistadas, como Memoria del Feminismo en el País Valenciano, 1970-1997, Entrevistas a mujeres vinculadas a Ca la Dona, Memoria 35 años, Entrevistas realizadas a mujeres del CIFFE, Haciendo historia. Género y Transición, Archivo de Fuentes Orales para la Historia Social de Asturias y Archivo Oral del Sindicalismo Socialista. Compuesto por fuentes, en algunos casos son las únicas que se conservan al objeto de componer un relato de lo sucedido, el relato biográfico de las protagonistas es fundamental para contrastar información, indagar en otras fuentes historiográficas, hemerográficas y archivos.

\subsubsection{Proyecto Entrevistas realizadas a mujeres}

El proyecto Entrevistas realizadas a mujeres del Centro de Información y Formación Feminista (CIFFE) está dirigido por la Federación de Mujeres Flora Tristán y Federación Castellana Amas de Casa, que fundó el CIFFE para, además, salvaguardar la documentación impresa. Entre los años 1985 y 1987, realizaron un proyecto de fuentes orales, recopilando un total de 218 testimonios de activistas pertenecientes a distintas organizaciones de todo el territorio español. Actualmente, este caudal documental se conserva, junto a las transcripciones, en el Centro Documental de la Memoria Histórica, en Salamanca. El proyecto de memoria es esencial para conocer la historia del tardofranquismo y la transición en España, un trabajo instrumental para incorporar otros elementos para las historiografias en futuras investigaciones.

\subsubsection{Proyecto La Asamblea de Mujeres de Granada: 35 años de lucha feminista en la ciudad de Granada}

El proyecto La Asamblea de Mujeres de Granada: 35 años de lucha feminista en la ciudad de Granada es un proyecto de memoria oral de la asociación Asamblea de Mujeres de Granada, realizado en 2013, "con el objetivo final de editar un libro electrónico con texto, fotos y una película documental" (Asamblea Mujeres Granada, 2013) que recupere la historia feminista granadina. La presentación del proyecto se concreta en la web Memoria 35 años, haciendo visibles a las mujeres granadinas de la Asamblea de mujeres tales como: "Carmen Guerrero, Lola Hita, Pilar Palomo, Lola callejón, Pilar Merediz, Cristina Isasi, Ana Jerez, Socorro Robles, Maite Pavón Palacios, Maribel Guijarro, Kim Pérez, Emilia Barrio Rodríguez, María José Belbel, Paqui García Bullejos, Josefina Jiménez Betancur, Mercedes Belbel Bullejos, Conxa Pasarin Rua; Tránsito Fernández Habas y Lorena Saletti Cuesta del Grupo Tomakandela", entre otras, que han sido partícipes de las luchas feministas (Asamblea Mujeres Granada, 2013).

\subsubsection{Proyecto Entrevistas a mujeres vinculadas a la asociación Ca la Dona}

El proyecto barcelonés Entrevistas a mujeres vinculadas a Ca la Dona, del Grup d'Historia Oral de Ca la Dona iniciado en el año 1999, con el objeto de recuperar y hacer visible la memoria del feminismo catalán. El Grupo realizó entrevistas a mujeres afines a la asociación barcelonesa Ca la Dona, en tanto se trata de uno de los referentes simbólicos del movimiento feminista en Barcelona. Uno de los objetivos del Grup d'Historia Oral de Ca la Dona es impulsar la creación de un archivo feminista, para realizar estudios e investigaciones que ayuden a una reconstrucción histórica de la realidad. La concepción del archivo, para preservar la memoria, surgió a partir del encuentro feminista organizado por Xarxa Feminista, celebrado en Barcelona, bajo el título Trobada de dones a Catalunya, 2, 3 i 4 de juny de 2006. Forman parte del Grupo, entre otras: "Àngels Candela, Núria Casals, Teresa Guarro, Mercè Otero-Vidal, Montserrat Otero Vidal, Eli Tejero y Laura Torrabadella" (Grup d'Historia Oral de Ca la Dona, 2006).

\subsubsection{Proyecto Archivo Oral del Sindicalismo Socialista-Mujeres}

En torno a los proyectos de historia oral producidos desde los Sindicatos obreros, destaca el proyecto Archivo Oral del Sindicalismo Socialista, integrado en el Archivo Histórico de la Fundación Francisco Largo Caballero, las entrevistas han sido transcriptas de audio a texto. Las feministas vinculadas al sindicato de la Unión General de Trabajadores, (UGT) que forman parte del proyecto de historia oral son: Matilde Fernández Sanz, Ana $\mathrm{M}^{\mathrm{a}}$. Ruiz-Tagle Morales, Josefina Arrillaga Lansorena, Alicia Ayala Velasco, Ángeles Díaz Samperio, Antonia Gárate Fernández, Ludivina García Arias, Carmen Muriana Ramírez, Aurora Roa Lara, Alejandra Soler Gilabert, Elena Vázquez Menéndez, Isabel Basterra Tur, Luisa Fernández Lafuente, Josefina Vidal Morera y 
Teresa Sanz Yáñez (Rodríguez Salvanés, Colsa Aguirre y Garay Gutiérrez, s.f.), rescatando las historias vitales como mujeres y sindicalistas militantes y dirigentes de la UGT.

\subsubsection{Proyecto Biografías obreras y militancia sindical en las Comisiones Obreras}

La colección Biografías obreras y militancia sindical en las Comisiones Obreras, CC.OO., basada en testimonios audiovisuales de sindicalistas, fue elaborada entre los años 2002 y 2012, complementando las fuentes orales de la Red de Archivos de CC.OO. De las 111 entrevistas, solo 34 son mujeres, representando un $31 \%$ de la colección, la colección Biografías obreras y militancia sindical en las CC.OO. es una fuente oral complementaria a las existentes en la Red de Archivos de Comisiones Obreras, que incluyen testimonios de varios territorios como Cataluña, Comunitat Valenciana, Andalucía y Galicia. A saber, los testimonios de mujeres se centran más en el Movimiento Democrático de Mujeres, que en las sindicalistas de las Comisiones Obreras. Entre las feministas entrevistadas se encuentran activistas como Cristina Almeida, Mercedes Comabella, Begoña San José y Manuela Carmena. Este proyecto se encuentra catalogado y disponible en el Archivo de Historia del Trabajo (AHT) de la Fundación $1^{\circ}$ de Mayo. El proyecto estuvo autofinanciado por la Confederación Sindical de CC.OO. (Archivo Historia del Trabajo, s.f.).

\subsubsection{Proyecto Archivo de Fuentes Orales para la Historia Social de Asturias}

El proyecto Archivo de Fuentes Orales para la Historia Social de Asturias (AFOHSA), adscrito a la Universidad de Oviedo, "promueve la recopilación, conservación y difusión del patrimonio oral de Asturias, con la finalidad de acercar tanto a investigadores como interesados un rico caudal de informaciones almacenado en las memorias y experiencias de los hombres y mujeres protagonistas de nuestro pasado reciente" (Memoria Social de Asturias, 2010). El proyecto AFOHSA comprende un número importante de "entrevistas, relatos de vida, testimonios de represión y de violencia política en Asturias relativos a las huelgas de 1962" y las "culturas del trabajo" y otros temas afines a mujeres y hombres. "A la espera de su archivo, la única serie que por el momento se encuentra libre para su consulta es la denominada Historias de Vida" (Memoria Social de Asturias, 2010).

Los relatos de las historias de vida del proyecto Archivo de Fuentes Orales para la Historia Social de Asturias (AFOHSA) son los testimonios orales de las mujeres como Anita Sirgo Suárez (PCE) y Celestina Marrón Llaneza (PCE), represaliadas y torturadas en las huelgas mineras de 1962: Blanca Huarte Erviti (PCE), María Ana Martínez Campa (Maruja) (PCE), Azucena González Uría, Encarnación Álvarez Fernández (PCE), Alvarina Rodríguez Vallina (PCE), Araceli Ruiz Toribios, (PCE), Águeda Ruiz Toribios (JSU y PCE), Laura González Álvarez (PCE, IU), Carmen Garrido González (HOAC), Aída Fuentes Concheso (PSOE, JOC, USO). Todas las mujeres entrevistadas son luchadoras en la clandestinidad en un período en el que los sindicatos y partidos políticos eran ilegales.

\subsubsection{Proyecto Memoria del Feminismo en el País Valenciano, 1970-1997}

El proyecto documental Memoria del Feminismo en el País Valenciano, 1970-1997, desarrollado en la Universitat de València (2011), por las investigadoras Concepción Gisbert Jordá, Dolores Sánchez Durá y Má. Teresa Yeves Bou, vinculadas al Institut Universitari d'Estudis de la Dona, financiado por el Instituto de la Mujer (Memoria del feminismo en el País Valenciano, 2012), rescata testimonios de las feministas valencianas en la transición. El proyecto es un estudio sociológico e histórico, cuyo objetivo central es "recuperar la memoria de las mujeres del movimiento feminista en el País Valenciano y sobre el proceso de toma de conciencia individual y colectiva" (Memoria del feminismo en el País Valenciano, 2012), el proceso de transformación en la evolución de las mujeres feministas "en sus relaciones con en vida pública, en sus diferentes opciones vitales", así como en la vida privada. En la década de los años ochenta surgen nuevos grupos feministas como Mujeres y Salud, Mujeres de Negro, Comissió 8 de març y Federación de Mujeres Progresistas del País Valencià, entre otros.

El proyecto Memoria del Feminismo en el País Valenciano, 1970-1997 es accesible en la web Feministas Valencianas, incluye las grabaciones de entrevistas a 26 feministas valencianas representativas de diferentes etapas, feminismos y diferentes ámbitos territoriales de la Comunidad Valenciana. Todas ellas participaron en el movimiento feminista en el País Valenciano durante el periodo de los años 1970-1997 como: "Ana Rodríguez (conocida como Ana Cari), perteneció al Movimiento Democrático de las Mujeres y a la Comisión de la Mujer del Ateneo Mercantil de Valencia, Trini Simó, Olga Quiñones, Inmaculada Garín, Elvira Vázquez y Carmen Alborch (política, escritora), todas ellas pertenecientes a Asociación de Mujeres Universitarias de Valencia, esta última junto a Isabel Robles, Consuelo Catalá, Isabel Morant (profesora), Luisa Moltó, Gusi Bertomeu, Amelia Abarca, de Asamblea de Mujeres de Valencia; Dolors Juan, del Grupo Terra; Llum Quiñonero, de Grup de Dones de Alacant; y Asunción Ventura Franch, de Col.lectiu Feminista de Castellón" (Feministas Valencianas, 2011). 


\subsubsection{Proyecto Una Transición en femenino}

El proyecto audiovisual Una Transición en femenino, de la Universidad de Zaragoza, desarrollado por un grupo de historiadoras en el año 2013, es una investigación sobre las mujeres zaragozanas en la transición y de su protagonismo en la historia. Su objetivo es visibilizar a las protagonistas de ese periodo, a través del relato testimonial de feministas zaragozanas como Irene Abad, Amparo Bella, Julia Casanova, Ángela Cenarro, Gloria Labarta, Rosa Fernández y Concha Rodríguez, en el que se reflexiona sobre la realidad social y política específica que vivieron las mujeres en el período de la transición (Universidad de Zaragoza, 2013).

\subsubsection{Proyecto Haciendo historia: Género y Transición}

El proyecto Haciendo historia: Género y Transición, adscrito al Centro de Estudios sobre la Mujer de la Universitat d'Alacant, es un proyecto, en cuyo equipo de investigadoras participan las profesoras de universidad Carmen Mañas, Mar Esquembre y Mónica Seco, dirigido por la investigadora Nieves Montesinos Montesinos. En él se recopilan los testimonios y documentos que, conjuntamente, visibilizan la participación de las mujeres en la transición. La web del proyecto contiene los documentos y transcripciones de las entrevistas en vídeo e imágenes de participantes como Carmen Alberola Rodríguez, Cristina Almeida Castro, Margarita Borja, Concha Collado Mateo, Merche Comabella, Asunción Cruañes Molina, Ángeles Espí Silvestre, Lidia Falcón O’Neill, Carmen García Santos, Alicia Gónzalez Beltrán, Helia Gónzalez Beltrán, Concha Jarabo Barruetabeña, Rafaela Mira Carratalá, Justa Montero Corominas, Marina Olcina González, Carmen Pacheco Martínez, Ana María Pérez del Campo Noriega, Encarna Pérez Guardiola, Llum Quiñonero Hernández, Silvia Ramírez Pacheco, $M^{\mathrm{a}}$. Teresa Revilla López, Mª . Pilar Rodríguez Clavel, Berta Saiz Cáceres, Rosalía Sanjuan Ayelo, Francisca Sauquillo Pérez del Arco, Rosalía Sender Begué, Nieves Simón Rodríguez, Marina Subirats Martori, Luisa Vidal Gonzálbez, Victoria Virtudes Fernández; y la exposición itinerante nombrada Mujeres de la transición (Haciendo historia. Género y Transición, 2014).

\subsubsection{Proyecto Mujer y Memoria}

El proyecto Mujer y Memoria es un espacio digital ideado por Aranzazu Borrachero, profesora de la Universidad de la Ciudad de Nueva York. Un sitio web creado para albergar proyectos que recuperen y difundan la memoria histórica de las mujeres españolas del siglo XX. El primero de los proyectos realizados, Madres e Hijas de la Transición Española, es un archivo audiovisual que recoge, preserva y presenta de forma interactiva relatos de vida de mujeres españolas que se hicieron adultas y madres durante el franquismo. Las hijas, cuyas vidas han transcurrido, en su mayor parte, durante la Transición y la democracia, entrevistan a sus madres. Y el segundo proyecto, Maternidades robadas, recopila testimonios audiovisuales y documentación sobre "robos de recién nacidos" en hospitales públicos y privados de España durante la dictadura y los primeros años de democracia. En las grabaciones, las víctimas de los "bebés robados" madres, padres, hermanas, hermanos, hijos e hijas narran su continua búsqueda, detallan sus denuncias y peticiones de investigación, y explican su trabajo de activismo social y político, en la búsqueda de justicia y reparación del daño (Mujer y Memoria, s.f.).

\subsection{Proyectos digitales para el estudio del movimiento feminista de la transición}

A finales de esta segunda década del siglo XXI son escasos los proyectos de digitalización documental como nuevas fuentes digitales para transmitir la historia, a diferencia de los proyectos de digitalización documental referidos a la historia de las mujeres y del feminismo en el extranjero, que se vinculan a las universidades. Los proyectos estudiados por Codina-Canet en la Universidad d'Angers, Harvard University. Smith College, University of Nebraska-Lincoln's. Indiana University, University of Iowa, Universty of San Diego, Duke University, Universität Osna-brück, Universität Düsseldorf son proyectos locales consolidados y persistentes en el tiempo para preservar los fondos documentales históricos de organizaciones y activistas feministas. Muchos son proyectos que comprenden la digitalización y acceso virtual al documento. En cambio, en España con respecto a los proyectos de mencionados, existe un retraso considerable (Codina Canet, 2019: 333).

El Centro de documentación Maite Albiz, en Bilbao; el Centro de Documentación - Biblioteca de Mujeres del Instituto Promoción de Estudios Sociales (IPES), en Navarra; la Biblioteca de Mujeres, en Madrid; y el proyecto del Museo de Arte Contemporáneo de Castilla y León (MUSAC) han iniciado ya, de forma específica, proyectos digitales de documentación histórica sobre feminismos y movimientos de mujeres.

\subsubsection{Proyecto Centro de documentación digital Maite Albiz}

El Centro de documentación Maite Albiz en Bilbao, en el cual se conservan los documentos históricos correspondientes a la Asamblea de Mujeres de Bizkaia, ha iniciado un proyecto de documentación digital, 
estructurándose el archivo digital en cuatro tipologías documentales: Jornadas, Revistas, Carteles y una cuarta miscelánea de documentos históricos compuesta por más de quinientos documentos y algunas publicaciones periódicas o revistas. Contiene la recopilación de las ponencias y actas de jornadas feministas que se han celebrado por toda la geografía española, en este período, accesibles desde el archivo digital (Centro de Documentación de Mujeres, 2007-2011).

\subsubsection{Proyecto Recuperación, preservación y difusión del legado histórico documental del movimiento feminista y de mujeres}

El Centro de Documentación-Biblioteca de Mujeres del IPES dinamizaron, desde el año 2015, el proyecto Recuperación, preservación y difusión del legado histórico documental del movimiento feminista y de mujeres en Navarra, con el objeto de recuperar y preservar el legado histórico documental de las mujeres de Navarra, para que esté accesible a toda la población (Biblioteca de Mujeres. Fundación IPES Elkartea, 2016) ya se han digitalizado documentos históricos, investigaciones pioneras e inéditas en Navarra y documentos descatalogados, fuentes para la reflexión y evolución del movimiento feminista y con el avance en derechos de las mujeres en Navarra. Posiblemente, son aproximadamente 500 documentos digitalizados de diversa tipología de documentación digitalizada, ya que se compone de folletos, panfletos, afiches o pegatinas, textos de debate y ponencias en organizaciones feministas, secciones de mujeres en partidos políticos y sindicatos, en jornadas feministas, en conferencias y congresos de partidos políticos sobre feminismos, movimiento de mujeres, textos sobre proyectos de leyes, textos legales y libros. La documentación recogida abarca los años 70 y 80 y, en menor medida, los 90; que conforman la biblioteca digital.

\subsubsection{Proyecto Archivo de Feminismos de León}

Igualmente, el proyecto digital Archivo de Feminismos de León es un proyecto colaborativo entre asociaciones feministas organizado por el Museo de Arte Contemporáneo de Castilla y León (MUSAC). Se trata de un proyecto digital de una colección virtual genealógica del movimiento feminista y de los distintos grupos feministas de la ciudad, tales como: las asociaciones leonesas Adavas, Flora Tristán, Insumisas al patriarcado, Isadora Duncan, Líneas de Fuga, Mujer y Revolución, PELOS y Asociación Simone de Beauvoir participan en el proyecto, aportando documentación privada e incluye una colección de carteles digitalizados desde el año 1977 (Archivo de Feminismos de León, 2018). Otra sección aporta documentación de las acciones en espacio públicos de la ciudad de León, el "Docu-Acciones". En León han emprendido un trabajo de recuperación de la documentación que podría derivar y encauzarse en un proyecto de archivo digital de envergadura.

\subsubsection{Proyecto Carteles de la Biblioteca de Mujeres}

Es un proyecto de digitalización de la colección de más de 2.000 carteles que custodia la Biblioteca de Mujeres en Madrid. Se agrupan los carteles en diecisiete temas: 8 de Marzo, Aborto, Arte, Bibliotecas y Centros de documentación, Calendarios, Deportes, Ocio, Derechos, Economía, Trabajo, Educación, Feminismo, Lesbianismo, Literatura, Otros países, Política, Salud, Sociedad y Violencia. Se accede a una descripción catalográfica de cada cartel, se permite navegar y descargar los carteles (Biblioteca de Mujeres, 2019).

\subsubsection{Proyecto Archivo de la Transición}

Se trata de un proyecto digital de la Asociación de Memoria Histórica del partido del Trabajo de España. Desde el apartado Movimientos sociales-Mujeres se accede a varios tipos de documentos históricos como estatutos, programas de actividades, revistas editadas por los grupos, cartas, campañas, comunicados de prensa, entre otros. Algunos de los grupos y asociaciones que han cedido algunos documentos son: Movimiento Democrático de Mujeres, Asociaciones de Amas de Casa, Associacions de Mestresses de Casa, Coordinadora Estatal de Asoc. de Amas de Casa, Plataforma de Org. de Mujeres de Madrid, Unión de Mujeres Antifascistas, Unión de Mujeres Republicanas Revolucionarias, Unión Popular de Mujeres y Organización de Mujeres Demócratas Archivo de la Transición. (2020).

En resumen, la siguiente tabla concentra los proyectos documentales citados para la reconstrucción de la Historia de las mujeres. 
Tabla 1. proyectos documentales para la reconstrucción de la Historia de las mujeres

\begin{tabular}{|c|c|c|}
\hline \multicolumn{3}{|c|}{ PROYECTOS DOCUMENTALES DE MEMORIA E HISTORIA DE LAS MUJERES } \\
\hline Nombre del proyecto & Entidad responsable & URL \\
\hline $\begin{array}{l}\text { Proyectos del Seminario de } \\
\text { Fuentes Orales }\end{array}$ & $\begin{array}{l}\text { Depart. Historia Contempo- } \\
\text { ránea. Universidad Complu- } \\
\text { tense }\end{array}$ & $\begin{array}{l}\text { https://web.archive. org/web/20161105124016/ } \\
\text { http://seminariofuentesorales.es/proyectos.html }\end{array}$ \\
\hline $\begin{array}{l}\text { Proyectos de la Universidad } \\
\text { Nacional de Educación a } \\
\text { Distancia }\end{array}$ & $\begin{array}{l}\text { Universidad Nacional de } \\
\text { Educación a Distancia }\end{array}$ & $\begin{array}{l}\text { https://canal.uned.es/series/5a6f3821b1111fb- } \\
\text { d3a8b4569 } \\
\text { https://canal.uned.es/serial/index/id/1362 } \\
\text { https://canal.uned.es/serial/index/id/1361 }\end{array}$ \\
\hline Diccionari Biogràfic de Dones & $\begin{array}{l}\text { Centro de Investigación } \\
\text { de Mujeres. Universitat de } \\
\text { Barcelona }\end{array}$ & $\begin{array}{l}\text { https://web.archive.org/web/20150812223127/http:// } \\
\text { dbd.cat/index.php?option=com_content\&view=arti- } \\
\text { cle\&id=1\&Itemid=6 }\end{array}$ \\
\hline Álbum de Mulleres & $\begin{array}{l}\text { Comisión de Igualdad del } \\
\text { Consejo de Cultura Gallega }\end{array}$ & $\begin{array}{l}\text { http://culturagalega.gal/album/detalle_especial. } \\
\text { php?id=129 }\end{array}$ \\
\hline \multicolumn{3}{|c|}{ PROYECTOS DE MEMORIA ORAL Y DIGITAL SOBRE FEMINISMO ORGANIZADO EN LA TRANSICIÓN } \\
\hline Nombre del proyecto & Entidad responsable & URL \\
\hline $\begin{array}{l}\text { Entrevistas realizadas a mu- } \\
\text { jeres }\end{array}$ & $\begin{array}{l}\text { Centro de Información y } \\
\text { Formación Feminista }\end{array}$ & \\
\hline $\begin{array}{l}\text { La Asamblea de Mujeres de } \\
\text { Granada: } 35 \text { años de lucha } \\
\text { feminista en la ciudad de } \\
\text { Granada }\end{array}$ & $\begin{array}{l}\text { Asociación Asamblea de } \\
\text { Mujeres de Granada }\end{array}$ & http://35amg.blogspot.com/ \\
\hline $\begin{array}{l}\text { Entrevistas a mujeres vincula- } \\
\text { das a la asociación Ca la Dona }\end{array}$ & $\begin{array}{l}\text { Grup d'Historia Oral de Ca } \\
\text { la Dona }\end{array}$ & $\begin{array}{l}\text { https://web.archive.org/web/20190516120524/http:// } \\
\text { www.caladona.org/grups/?page_id=128 }\end{array}$ \\
\hline $\begin{array}{l}\text { Archivo Oral del Sindicalismo } \\
\text { Socialista-Mujeres }\end{array}$ & $\begin{array}{l}\text { Fundación Francisco Largo } \\
\text { Caballero }\end{array}$ & $\begin{array}{l}\text { http://portal.ugt.org/fflc/hemeroteca/hemeroteca00. } \\
\text { htm }\end{array}$ \\
\hline $\begin{array}{l}\text { Biografías obreras y militancia } \\
\text { sindical en las Comisiones } \\
\text { Obreras }\end{array}$ & Fundación $1^{\circ}$ de Mayo & $\begin{array}{l}\text { http://www.1mayo.ccoo.es/Archivo_Historia_del_ } \\
\text { Trabajo }\end{array}$ \\
\hline $\begin{array}{l}\text { Archivo de Fuentes Orales para } \\
\text { la Historia Social de Asturias }\end{array}$ & Universidad de Oviedo & https://www.unioviedo.es/AFOHSA/ \\
\hline $\begin{array}{l}\text { Memoria del Feminismo en el } \\
\text { País Valenciano, 1970-1997 }\end{array}$ & $\begin{array}{l}\text { Institut Universitari d'Estudis } \\
\text { de la Dona. Universitat de } \\
\text { Valencia }\end{array}$ & https://feministasvalencianas.wordpress.com/ \\
\hline Una Transición en femenino & Universidad de Zaragoza & https://vimeo.com/109454690 \\
\hline $\begin{array}{l}\text { Haciendo historia: Género y } \\
\text { Transición }\end{array}$ & $\begin{array}{l}\text { Centro de Estudios sobre la } \\
\text { Mujer. Universitat d'Alacant }\end{array}$ & http://www.mujeresytransicion.es \\
\hline Mujer y Memoria & $\begin{array}{l}\text { Aranzazu Borrachero. } \\
\text { Universidad de la Ciudad de } \\
\text { Nueva York }\end{array}$ & https://mujerymemoria.org/web/ \\
\hline \multicolumn{3}{|c|}{ PROYECTOS DE DIGITALES PARA EL ESTUDIO DEL MOVIMIENTO FEMINISTA DE LA TRANSICIÓN } \\
\hline Nombre del proyecto & Entidad responsable & URL \\
\hline $\begin{array}{l}\text { Centro de documentación digi- } \\
\text { tal Maite Albiz }\end{array}$ & $\begin{array}{l}\text { Centro de documentación } \\
\text { Maite Albiz }\end{array}$ & https://emakumeak.org/web/archivo_digital \\
\hline $\begin{array}{l}\text { Recuperación, preservación y } \\
\text { difusión del legado histórico } \\
\text { documental del movimiento } \\
\text { feminista y de mujeres }\end{array}$ & $\begin{array}{l}\text { Biblioteca de Mujeres. Fun- } \\
\text { dación IPES }\end{array}$ & $\begin{array}{l}\text { http://www.ipesmujeres.org/biblioteca/documenta- } \\
\text { cion-historica-digitalizada }\end{array}$ \\
\hline $\begin{array}{l}\text { Archivo de Feminismos de } \\
\text { León }\end{array}$ & $\begin{array}{l}\text { Museo de Arte Moderno de } \\
\text { Castilla y León }\end{array}$ & http://www.archivofeminismosleon.org \\
\hline $\begin{array}{l}\text { Carteles de la Biblioteca de } \\
\text { Mujeres }\end{array}$ & Biblioteca de Mujeres & $\begin{array}{l}\text { https://www.mujerpalabra.net/bibliotecademujeres/ } \\
\text { pages/carteles/index.htm }\end{array}$ \\
\hline Archivo de la Transición & $\begin{array}{l}\text { Asociación de Memoria His- } \\
\text { tórica del partido del Trabajo } \\
\text { de España }\end{array}$ & $\begin{array}{l}\text { https://archivodelatransicion.es/archivo-organizacio- } \\
\text { nes/movimientos-sociales-mujeres }\end{array}$ \\
\hline
\end{tabular}




\section{Conclusiones}

Los proyectos recogidos en la investigación muestran nuevas formas de transmitir, de comunicar la historia. Y proyectos sobre los testimonios de mujeres de historia oral para la recuperación de la memoria de las mujeres que participaron en el proceso político de España durante la Transición española, han surgido en algunas Comunidades Autónomas.

1. Entre los principales proyectos de preservación documental, a partir del análisis historiográfico, muchos se caracterizan por la creación de nuevas fuentes para futuras investigaciones.

2. Los proyectos de memoria histórica, fundamentalmente, crean fuentes orales, que testimonian los hechos históricos mediante relatos de vida de las mujeres entrevistadas.

3. Los proyectos recopilados en un número muy elevado están vinculados a las universidades, sin embargo, muchos de ellos carecen de continuidad en el tiempo.

4. Los proyectos documentales de digitalización, que poseen continuidad en el tiempo, se realizan en instituciones públicas, como son el Ảlbum de Mulleres del Consello de la Muller en Galicia y el Archivo de Feminismos de León del Museo de Arte Contemporáneo de Castilla y León.

5. Y, también, poseen persistencia en el tiempo, aquellos otros proyectos de digitalización que surgen en los centros de documentación y bibliotecas de mujeres más consolidados, fruto de las propias asociaciones feministas como la Asamblea de mujeres de Bilbao del Centro de documentación Maite Albiz, el Centro de documentación-Bibliotecas de Mujeres del IPES, en Navarra y, la asociación Ca la Dona, en Barcelona.

6. Se concluye que existe un manifiesto abandono en la recuperación de archivos del feminismo en España, ya que se encuentra pendiente de realizar un proyecto de Centro de archivos del Feminismo que recupere el caudal documental de las organizaciones feministas privadas, que se digitalice la documentación, y que se concrete como una nueva forma de transmitir el pasado disponible para la investigación y para la ciudadanía.

El rescate del patrimonio documental de las organizaciones feministas de la Transición española es una tarea pendiente. Su estudio y preservación allanará el camino pendiente de revisión histórica del movimiento feminista en esta etapa, posibilitando una historiografía deconstruccionista, para recuperar la otra memoria basada en la función política desempeñada por las organizaciones de mujeres en el ámbito público durante la Transición política a la democracia.

\section{Referencias bibliográficas}

Alberch, R. ; Cruz-Mundet, J. R. (1999). ¡Archivese! Los documentos del poder El poder de los documentos. Madrid: Alianza Editorial.

Álbum de mulleres. (s.f.a). Aborto. Corenta anos de mobilizacións [en línea]. Disponible en: http://culturagalega.gal/ album/visor-pdf/aborto-corenta-anos-de-mobilizacions381web2.pdf

Codina-Canet, Ma . A. ; San Segundo, R. (2016). "Propuesta de un Centro de Archivo del Feminismo tras el análisis de los fondos documentales del movimiento feminista". Revista Española de Documentación Científica, 39 (1: e117). https://doi.org/10.3989/redc.2016.1.1245

Codina-Canet, Ma. A. (2019). Fundamentación de Centro de Archivo de memoria del Movimiento Feminista [Tesis doctoral inédita]. Getafe: Universidad Carlos III de Madrid.

Biblioteca de Mujeres. Fundación IPES Elkartea. (2016). Proyecto "Recuperación, preservación y difusión del legado histórico documental del movimiento feminista y de mujeres en Navarra" [en línea]. Disponible en: http://www. ipesmujeres.org/pdf/pdf-memoria-digitalizacion-2016.pdf

Egido-León, A. ; Eiroa-San Francisco, M. (2017). "Redes sociales, historia y memoria digital de la represión de mujeres en el Franquismo". Revista de historiografia, 27, pp. 341-361. Disponible en: https://e-revistas.uc3m.es/index.php/ REVHISTO/article/view/3977/2505

González-Quintana, A. (2009). Principios básicos sobre el papel de los archivos en la defensa de los derechos humanos [en línea]. Disponible en: http://www.ica.org/sites/default/files/ReportGonzalez-QuintanaES.pdf

Memoria del feminismo en el País Valenciano. (2012). Disponible en: https://feministasva lencianas.files.wordpress. com/2012/10/feministas-valencianas.pdf

Pérez-Pais, C. ; Mariño-Costales, M. (2013). Recuperación e memoria documentación e memoria do movemento feminista organizado en Galicia [en línea]. Disponible en: http://consellodacultura.gal/mediateca/extras/CCG 2013 Recuperacion-da-documentacion-e-memoria-do-Movemento-Feminista-Organizado-en-Galicia.pdf

Rodríguez-Salvanés, J. ; Colsa-Aguirre, R. ; Gray-Gutiérrez, L. (s.f.). Archivo Oral del Sindicalismo Socialista (2007-2010) [en línea]. Disponible en: http://archivos.ugt.es/inventarios/ARCHIVO\%20ORAL\%20DEL\%20SINDICALISMO $\% 20$ SOCIALISTA.pdf 
Universidad Nacional de Educación a Distancia (UNED) (2013). Canal UNED. Historia crítica del Feminismo español (2) El Feminismo en la Segunda República [en línea]. Recuperado de https:/canal.uned.es/uploads/materials/resources/ pdf/1/8/1360862903381.pdf

\section{Documentos web}

Álbum de mulleres. (s.f.b). ¿que é o álbum de mulleres? [en línea]. Consejo da Cultura Galega. Comisión de Igualdade. Disponible en: http://culturagalega.gal/album/detalle_especial.php?id=129 [Fecha de consulta: 1/06/2019].

Archivo de Feminismos de León. (2018). Archivo de Feminismos de León [en línea]. León: Museo de Arte Contemporáneo de Castilla y León, MUSAC. Disponible en: http:/www.archivofeminismosleon.org/ [Fecha de consulta: 17/12/2018].

Archivo de la Transición. (2020). Archivo por organizaciones movimientos sociales: mujeres. [en línea].Disponible en https://archivodelatransicion.es/archivo-organizaciones/movimientos-sociales-mujeres [Fecha de consulta: 28/05/2020].

Archivo Historia del Trabajo. (s.f.). AHT - Archivo de Historia del Trabajo [en línea]. Madrid: Fundación $1^{\circ}$ de Mayo [en línea]. Disponible en: http://www.1mayo.ccoo.es/Archivo_Historia_del_Trabajo [Fecha de consulta: 17/12/2018].

Asamblea Mujeres Granada. (21 octubre, 2013). Memoria 35 años AMG [Mensaje en un blog]. Disponible en: http://35amg. blogspot.com/ [Fecha de consulta: 17/12/2018].

Biblioteca de Mujeres. (2019). Carteles. [en línea]. Disponible en https://www.mujerpalabra.net/bibliotecademujeres/ pages/carteles/index.htm [Fecha de consulta: 28/05/2020].

Brancas, M. (16 abril, 2015). Hace falta un empujón más. La importancia de la Memoria histórica feminista [en línea]. Disponible en: http://www.feministas.org/la-importancia-de-la-memoria.html [Fecha de consulta: 17/12/2018].

Centro de Documentación de Mujeres. (2007-2011). Centro de documentación digital [en línea]. Disponible en: https:// emakumeak.org/web/archivo_digital [Fecha de consulta: 17/12/2018].

Feministas Valencianas. (2011). Historia del feminismo en la Comunidad Valenciana [en línea]. Valencia: Feministas Valencianas. Disponible en: https://feministasvalencianas.wordpress.com/ [Fecha de consulta: 9/9/2018].

Grup d' Historia Oral de Ca la Dona. (2006). L'altaveu de totes - el blog de Ca la Dona [Blog]. Disponible en: http://www. caladona.org/grups/?page_id=128 [Fecha de consulta: 17/12/2018].

Haciendo historia. Género y Transición. (2014). Bienvenida al Proyecto: "Haciendo historia: Género y Transición en España”[ en línea]. Alicante: Universidad de Alicante. Disponible en: http://www.mujeres ytransicion.es/pages/home. php [Fecha de consulta: 17/12/2018].

Memoria Social de Asturias. (2010). Archivo de Fuentes Orales para la Historia Social de Asturias [en línea]. Disponible en: https://www.unioviedo.es/AFOHSA/ [Fecha de consulta: 17/12/2018].

Mujer y Memoria.(s.f.). Madres e hijas de la Transición española. Maternidades robadas [en línea]. Disponible en: https:// mujerymemoria.org/web/ [Fecha de consulta: 27/01/2020].

Seminario de Fuentes Orales. (s.f.a). Constitución y fines del SFO [en línea]. Disponible en: https://web.archive.org/ web/20170626100813/http://www.seminariofuentesorales.es/ [Fecha de consulta: 17/12/2018].

Seminario de Fuentes Orales. (s.f.b). Proyectos [en línea]. Disponible en: https://web.archive. org/web/20161105124016/ http://seminariofuentesorales.es/proyectos.html [Fecha de consulta: 17/12/2018].

Universidad de Zaragoza. (7 marzo, 2013). Una transición en femenino [Archivo de video]. Disponible en: https://vimeo. com/109454690 [Fecha de consulta: 9/9/2018].

Universidad Nacional de Educación a Distancia (UNED) (1998- 2017). Canal UNED. Derechos de la Mujer y equidad de género [Archivo de video]. Disponible en: https://ca nal.uned.es/serial/index/id/1362 [Fecha de consulta: 7/2/2019].

Universidad Nacional de Educación a Distancia (UNED) (1998). Canal UNED. Las mujeres en el mundo [Archivo de video]. Disponible en: https://canal.uned.es/serial/index/id/1262 [Fecha de consulta: 7/2/2019].

Universidad Nacional de Educación a Distancia (UNED) (2004). Canal UNED. Mujeres intelectuales. Mujeres [Archivo de video]. Disponible en: https://canal.uned.es/serial/index/id/1361 [Fecha de consulta: 7/2/2019].

Xarxa Vives de Universitats. (s.f.). Diccionari Biogràfic de Dones [en línea]. Disponible en: https://web.archive.org/ web/20150812223127/http://dbd.cat/index.php?option=com_content\&view=article\&id=1\&Itemid=6 [Fecha de la consulta: 18/12/2018]. 